\title{
MULTICOLOR PHOTOMETRY OF THE GALACTIC CLUSTER NGC 2362
}

\author{
CH. L. PER RY \\ Louisiana State University Observatory, Baton Rouge, La., U.S.A.
}

\begin{abstract}
U, B, V$ colors of CPD stars brighter than 12 mo in the central region of NGC 2362 . Color excess. Discussion of the distance modulus of the cluster.
\end{abstract}

\section{Introduction}

The galactic cluster NGC 2362 is a sparsely populated but tightly knit aggregate of early-type stars dominated by the fourth magnitude O-type star $\tau \mathrm{CMa}$. The cluster has been of considerable astronomical interest. Because it is only a few million years old, NGC 2362 has played a vital rôle in the extension of the $U B V$ zero-age main sequence toward the blue and bright end of the color-magnitude diagram; see, for example, an excellent discussion by Blaauw (1963), where references are given to earlier papers also involved with the development of the main-sequence fitting procedure. Likewise, the cluster will be an important addition in the development of the $u v b y$ and $\mathrm{H} \beta$ photometric systems. Recent observational investigations of the cluster include a photometric study by Johnson (1950) and a combined $U B V$ and MK classification study by Johnson and Morgan (1953). The present photometric investigation was undertaken because of both the intrinsic importance of the cluster and the variance in previous determinations of its distance.

\section{Observations}

All CPD stars brighter than $12^{\mathrm{m}} \cdot 0$ within an area of the sky between $7^{\mathrm{h}} 13^{\mathrm{m}} 00^{\mathrm{s}}$ and $7^{\mathrm{h}} 14^{\mathrm{m}} 20^{\mathrm{s}}$ (1875) of right ascension and between $-24^{\circ} 35^{\prime}$ and $-24^{\circ} 50^{\prime}$ of declination were chosen as the major part of the observing program. The four non-CPD stars brighter than $12 \mathrm{~m} .0$, as observed by Johnson and Morgan, completed the program. Additional $U B V$ observations were made to supplement the existing data. uvby and $\mathrm{H} \beta$ photometry was obtained for the majority of the stars in the immediate cluster region.

Details of the instrumentation, observing techniques, and reduction procedures have been described by Perry and Hill (1969). Table I lists the internal mean errors of a single observation. Henceforth, mean errors are quoted throughout the paper. Table II gives, in succession, the CPD numbers, the $U B V$, $u v b y$, and $\mathrm{H} \beta$ values obtained in this investigation, along with the number of nights on which observations of a given star were made; each star was usually observed twice during a given night. The $V$ magnitudes on the $U B V$ system, listed in the sixth column, were derived from the $y$ deflec- 
tions of the urby photometry. $U B V$ observations of the star $-24^{\circ} 2251$ were made but its photometric values are not included in Table II because its colors were outside the color range of the standard stars. It is a late-type object.

TABLE I

Internal mean errors of a single observation

\begin{tabular}{lccccccc}
\hline$V$ & $B-V$ & $U-B$ & $V$ & $b-y$ & $m_{1}$ & $c_{1}$ & $\beta$ \\
\hline $0 \div 019$ & $0 m 013$ & $0 \div 017$ & $0 \div 019$ & $0 m 013$ & $0 \div 017$ & $0 \div 019$ & $0 m 012$
\end{tabular}

The $U B V$ photometries of Johnson and Morgan and Table II were combined into a homogeneous system. The $V$ magnitudes derived from the $u v b y$ photometry were also included. Table III lists the CPD numbers, the Johnson numbers, the binary star numbers from the Index Catalogue of Visual Double Stars by Jeffers and van den Bos (1963), the final $U B V$ values, along with the number of observations. The MK spectral types, listed in the last column, are due to Johnson and Morgan or Schild (1970). The photometry for the four stars indicated in the fourth column of the table refers to the combined light of the binary components.

\section{Results}

\section{A. COLOR EXCESS OF NGC 2362}

The intrinsic colors of the program stars were determined by four methods, namely, (1) the $U B V Q$-method, (2) Strömgren's (1966) [u-b] calibration, (3) Crawford's (1970) $c_{0}$ calibration, and (4) MK spectral types. These methods will be discussed in some detail in turn.

The $U B V$ color indices listed in Table III were employed to compute the individual intrinsic color indices via the equations $(B-V)_{0}=0.335(U-B)-0.241(B-V)$ and $(U-B)_{0}=1.241(U-B)-0.893(B-V)$. The coefficients in the above relations are appropriate for a reddening slope in the $U B V$ color-color diagram of $E(U-B)$ / $E(B-V)=0.72$. A discussion of the $U B V Q$-method is given, for example, by Morgan and Harris (1956). Individual color excesses in both colors were next calculated via the equations $E(B-V)=(B-V)-(B-V)_{0}$ and $E(U-B)=(U-B)-(U-B)_{0}$. The twenty-nine program stars, selected as clusters members, yielded mean cluster color excesses of $E(B-V)=0.100 \pm 0^{\mathrm{m}} \cdot 020$ and $E(U-B)=0.070 \pm 0$ m.014. The questions of cluster membership is discussed in the next section.

Figure 1 illustrates the $U B V$ color-color diagram for NGC 2362 plotted with the data in Table III. The solid line traces the standard relation derived by Johnson and Morgan, shifted by 0.10 in $(B-V)$ and $0^{\mathrm{m}} .07$ in $(U-B)$. The small scatter about the standard relation indicates that the reddening is nearly uniform over the cluster region.

An ultraviolet color index for the uvby photometric system may be derived via the 


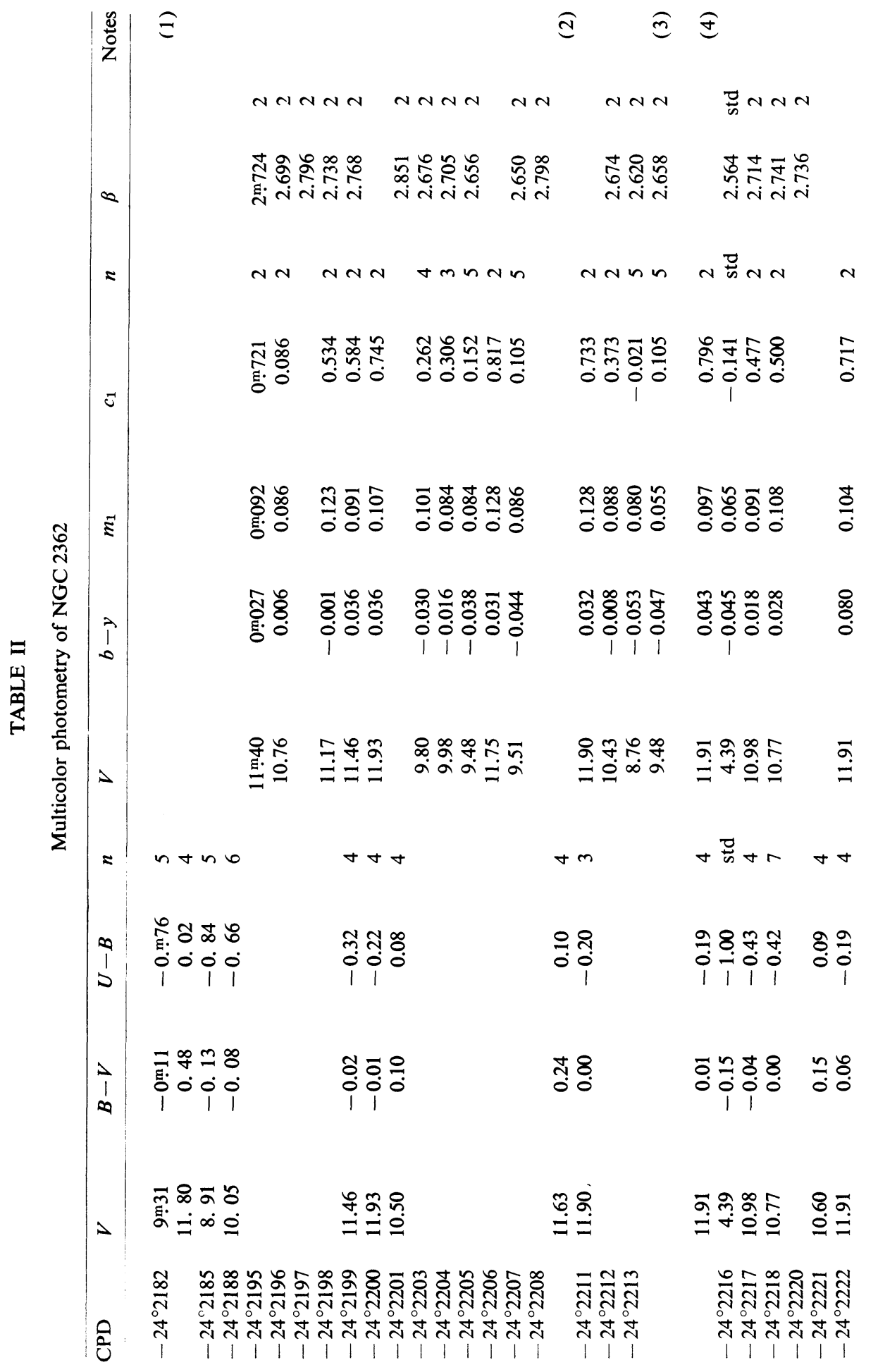


MULTICOLOR PHOTOMETRY OF THE GALACTIC CLUSTER NGC 2362

$\frac{\mathscr{E}}{\stackrel{0}{0}}$

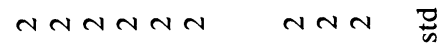

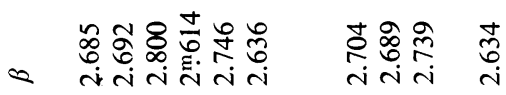

$=N m \operatorname{nnn} N \frac{\pi}{2}$

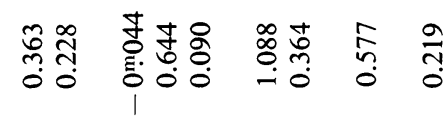

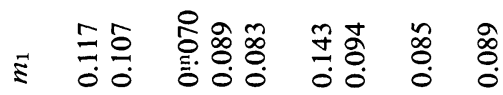

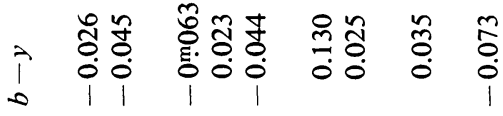

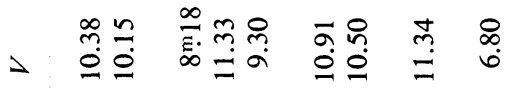

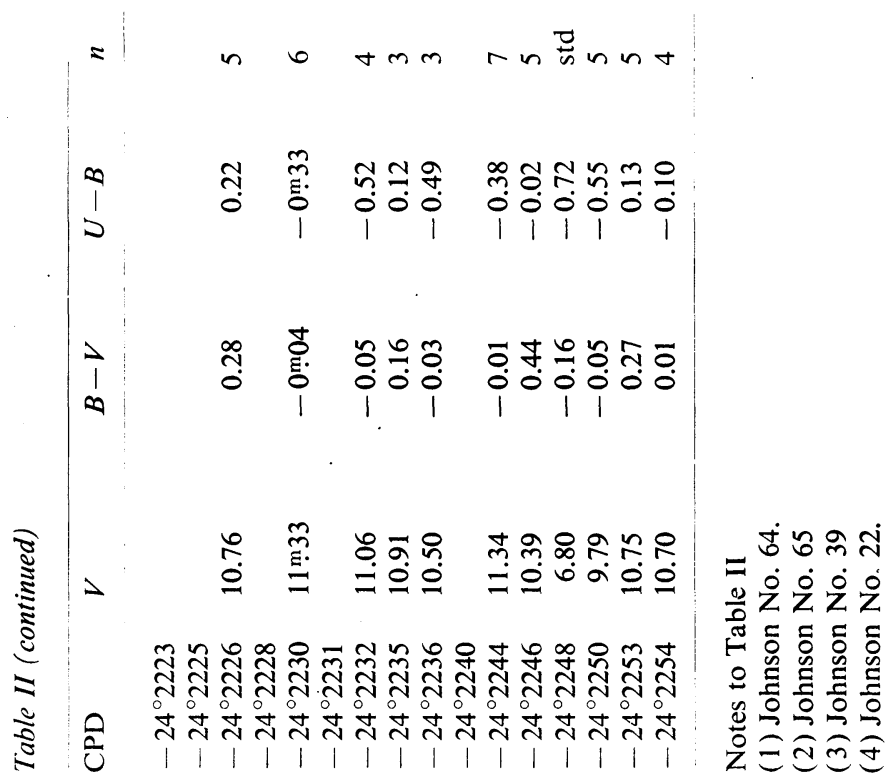


TABLE III

Combined $U B V$ photometry of NGC 2362

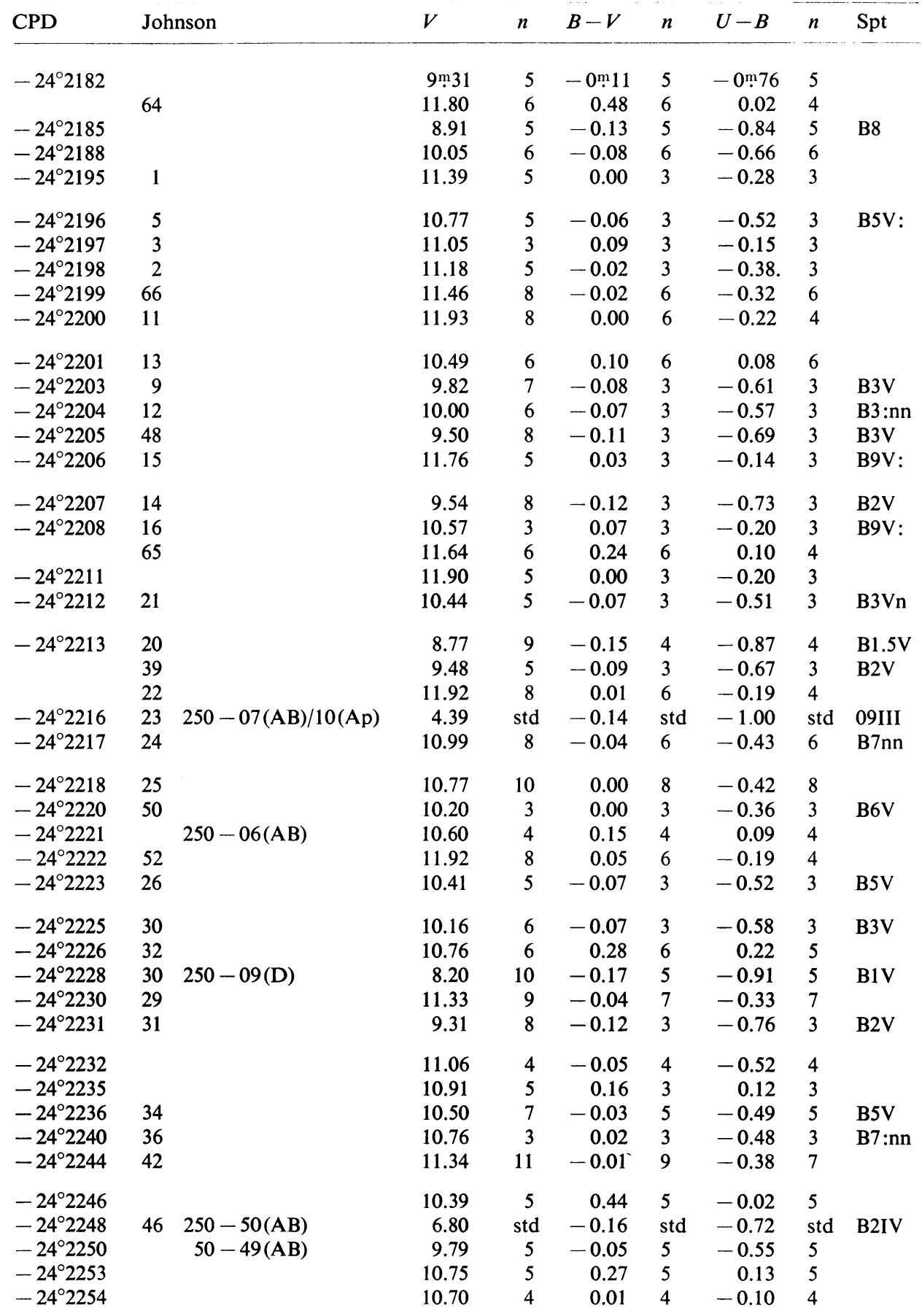




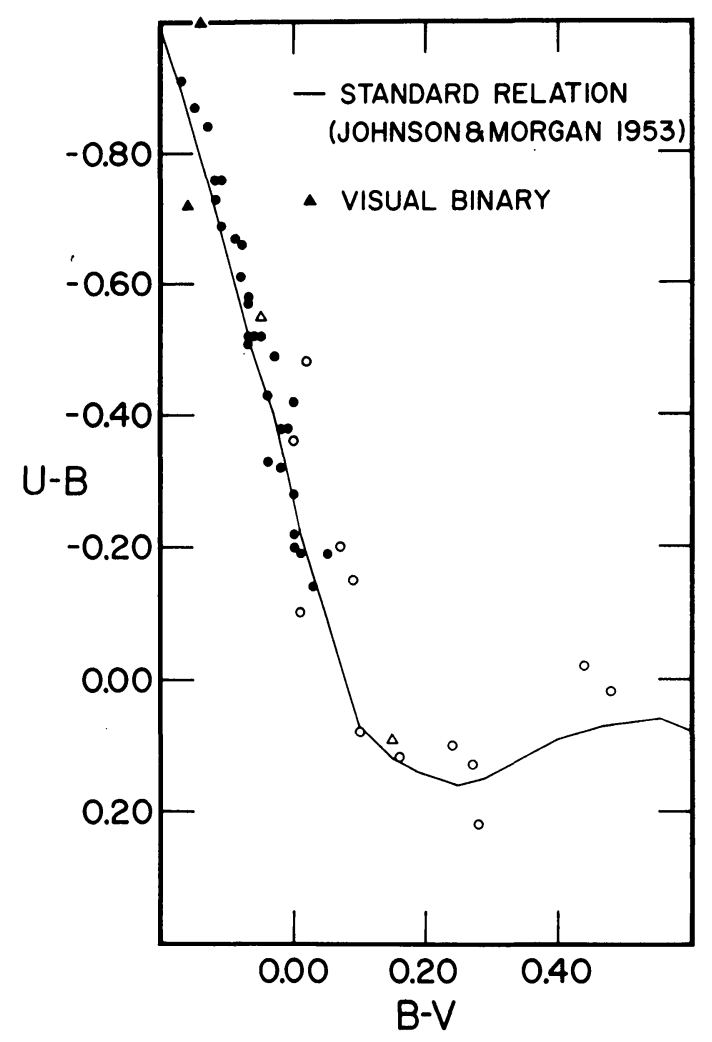

Fig. 1. The $U B V$ color-color diagram for NGC 2362. The solid line traces the standard relation of Johnson and Morgan (1953). Visual binaries are indicated.

equation $(u-b)=c_{1}+2 m_{1}+2(b-y)$. Strömgren (1966) has formed an index $[u-b]$ which is independent of interstellar reddening; the index is given by the equation $[u-b]=(u-b)-1.84(b-y)$. On the basis of $u v b y$ photometry of nearby and hence unreddened B stars, Strömgren found that $(b-y)_{0}$ is a function of $[u-b]$ only. After values of $(u-b)$ and $[u-b]$ were computed for those stars in Table II with uvby photometry, the intrinsic $(b-y)_{0}$ colors were interpolated from Strömgren's calibration. Individual color excesses were next calculated via the equation $E(b-y)=$ $(b-y)-(b-y)_{0}$. The nineteen program stars, selected as cluster members, yielded a mean cluster color excess of $E(b-y)=0 \cdot 090 \pm 0.022$. Crawford (1964) has shown that the color excesses in the $U B V$ and $u v b y$ photometric systems are related by the equation $E(B-V)=1.43 E(b-y)$; the above value of $E(b-y)$ is therefore equivalent to $E(B-V)=0^{\mathrm{m}} \cdot 129 \pm 0^{\mathrm{m}} \cdot 031$.

On the basis of uvby photometry of bright and hence unreddened B stars, Crawford (1970) has devised an iterative procedure to determine the intrinsic $(b-y)_{0}$ colors of early-type stars. One employs the observed $c_{1}$ values to obtain the first approximation to the $(b-y)_{0}$ colors via the equation $(b-y)_{0}=-0.116+0.097 c_{1}$. The color excesses 
are computed in the usual manner. Crawford (1966) has shown that the color excesses in $(b-y)$ and $c_{1}$ are related by the equation $E\left(c_{1}\right)=0.2 E(b-y)$. The observed $c_{1}$ values are corrected for reddening via the equation $c_{0}=c_{1}-E\left(c_{1}\right)$. The second approximation to the $(b-y)_{0}$ colors is obtained by substituting $c_{0}$ in place of $c_{1}$ in the first equation. One iteration was sufficient to calculate the individual $(b-y)_{0}$ colors and color excesses for those stars in Table II with uvby photometry. The twenty-three program stars, selected as cluster members, yielded a mean cluster color excess of $E(b-y)=0^{\mathrm{m}} .075 \pm 0^{\mathrm{m}} 015$. This value corresponds to $E(B-V)=0^{\mathrm{m}} \cdot 107 \pm 0^{\mathrm{m}} \cdot 021$.

The tabulation by Johnson (1963) of intrinsic $(B-V)_{0}$ and $(U-B)_{0}$ colors as a function of MK spectral type allows one to determine the $(B-V)_{0}$ colors for those program stars listed in Table III with MK spectral types. Individual color excesses were next computed. The fifteen program stars, selected as cluster members, yielded a mean cluster color excess of $E(B-V)=0{ }^{\mathrm{m}} \cdot 112 \pm 0$ m 022 .

\section{B. DISTANCE MODULUS OF NGC 2362}

The absolute magnitudes of the program stars were also determined by four methods, namely, (1) the intrinsic $(B-V)_{0}$ colors, (2) Fernie's (1965) $\left(\beta, M_{v}\right)$ calibration adopted by Strömgren (1966), (3) Crawford's (1970) $\left(\beta, M_{v}\right)$ calibration, and (4) MK spectral types. These methods will be discussed in some detail in turn.

In all four methods, the visual apparent magnitudes were corrected for interstellar absorption via either the equation $V_{0}=V-3.0 E(B-V)$, derived by Morgan, Harris, and Johnson (1953), or $V_{0}=V-4.3 E(b-y)$, derived by Crawford (1966).

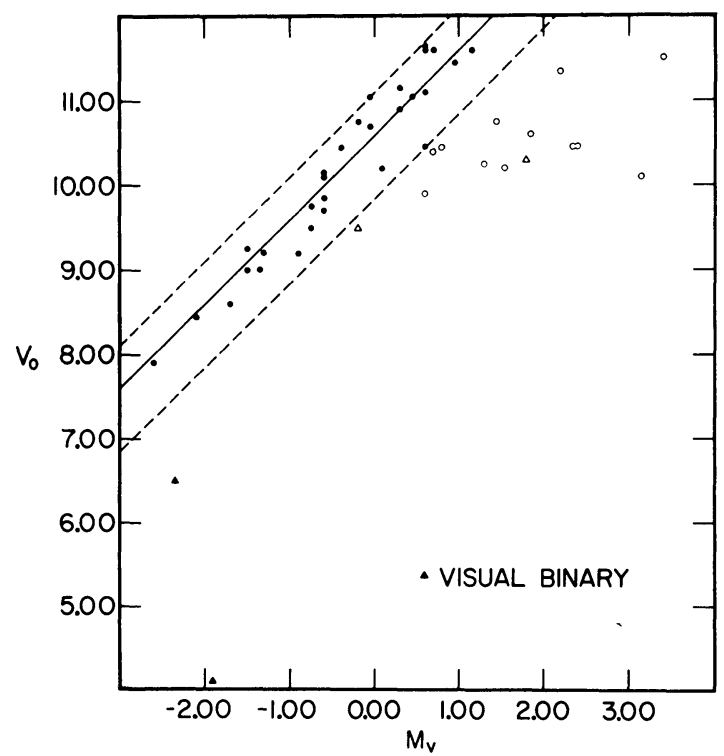

Fig. 2. The $U B V\left(V_{0}, M_{v}\right)$ diagram for NGC 2362. Probable cluster members are denoted by filled circles, non-members by open circles. Visual binaries are indicated. The dashed lines are discussed in the text. 
Following the example of Walker (1965), cluster membership was investigated by means of $\left(V_{0}, M_{v}\right)$ diagrams in all four methods. Figure 2 illustrates the $U B V\left(V_{0}, M_{v}\right)$ diagram for NGC 2362. Stars within the domain defined by the dashed lines are considered to be cluster members. These limits were set by assuming that no cluster member will lie more than 0.5 above the line $V_{0}=M_{v}+$ constant and that duplicity will not brighten a star more than 0.75 . The brightest members of a cluster may lie outside the membership domain becaúse they are in the process of evolving away from the main sequence.

Blaauw (1963) has tabulated visual absolute magnitude as a function of $(B-V)_{0}$ for the zero-age main sequence. The twenty-seven program stars, selected as cluster members, in Table III with $U B V$ photometry yielded a mean cluster distance modulus of $10^{\mathrm{m}} \cdot 60 \pm 0 \mathrm{~m} \cdot 26$.

Figure 3 illustrates the $U B V$ color-magnitude diagram for NGC 2362. The solid line traces Blaauw's ZAMS. The absolute magnitude scale on the right-hand side of the diagram is computed for a distance modulus of $10^{\mathrm{m}} \cdot 60$.

Strömgren (1966) adopted Fernie's (1965) $\left(\beta, M_{v}\right)$ calibration. The calibration was derived using various galactic clusters and associations with well-determined distance

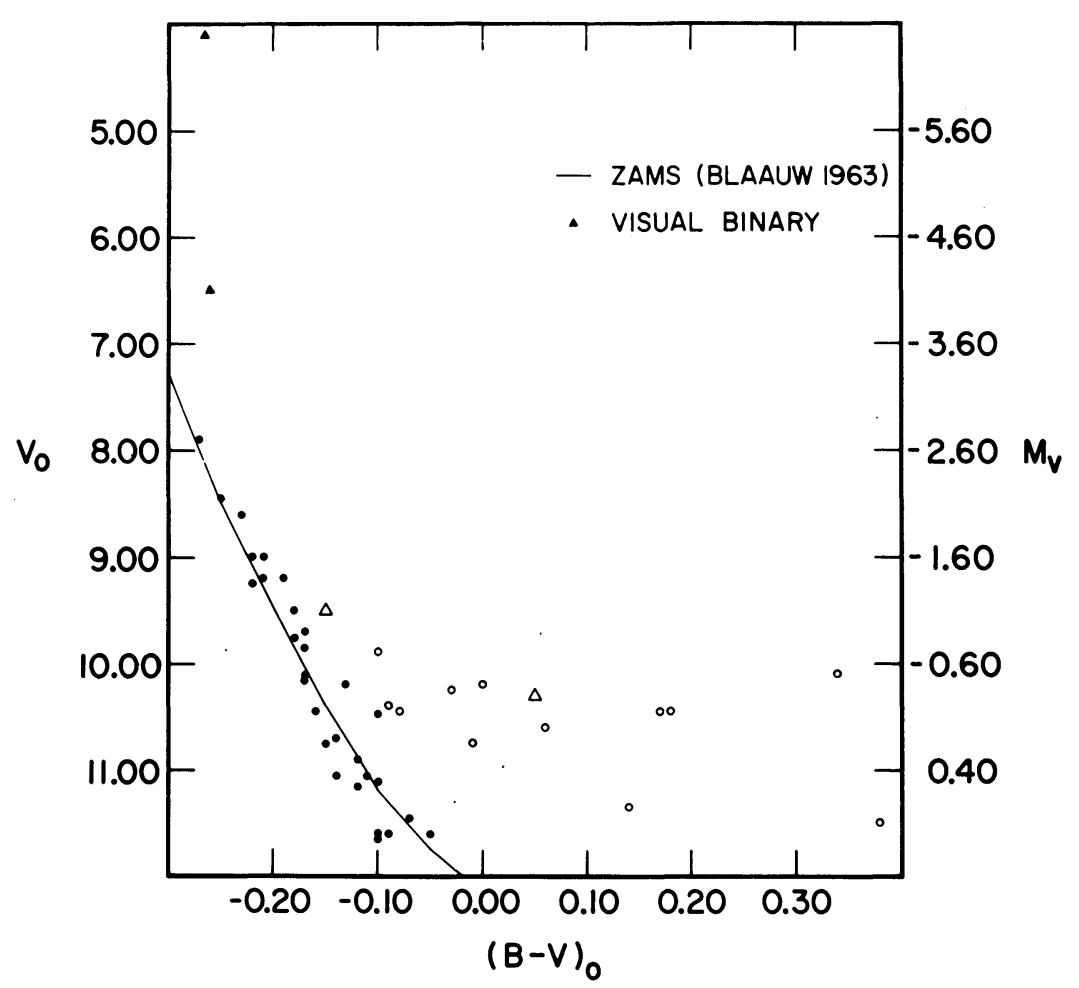

Fig. 3. The $U B V$ color-magnitude diagram for NGC 2362. Visual binaries are indicated. The solid line traces Blaauw's (1963) zero-age main sequence. The absolute magnitude scale on the right-hand side of the diagram is computed for a distance modulus of $10 \mathrm{~m} 60$. 
moduli; the cluster members had measured hydrogen line intensities which Fernie reduced to the photoelectric $\beta$ system. The nineteen program stars, selected as cluster members, in Table II with $\mathrm{H} \beta$ photometry yielded a mean cluster distance modulus of $11^{\mathrm{m}} \cdot 22 \pm 0^{\mathrm{m}} \cdot 24$.

Crawford (1970) derived his $\left(\beta, M_{v}\right)$ calibration by fitting the shape of the $\left(V_{0}, \beta\right)$ relations of various galactic clusters and associations; the zero point of the calibration was defined by the known distance moduli of the $\alpha$ Persei and Pleiades clusters. The eighteen program stars, selected as cluster members, in Table II with $\mathrm{H} \beta$ photometry yielded a mean cluster distance modulus of $11^{\mathrm{m}} \cdot 22 \pm 0.26$.

The tabulation by Blaauw (1963) of absolute magnitude as a function of MK spectral type also allows one to determine the absolute magnitudes of those stars in Table III with MK spectral types. The fifteen program stars, selected as cluster members, yielded a mean cluster distance modulus of $11^{\mathrm{m}} \cdot 27 \pm 0^{\mathrm{m}} \cdot 30$.

\section{Discussion}

Table IV includes a summary of the results from the four determinations of the mean cluster color excess and corrected distance modulus of NGC 2362 made in this paper. The adopted values are averages of the four determinations weighted according to their mean errors, with the following exception. Because the $\left(\beta, M_{v}\right)$ calibrations of Fernie and Crawford are quite similar, only one value of $V_{0}-M_{v}=11^{\mathrm{m}}$.22 was included in the weighted average. It should be pointed out that the color excess and distance modulus determined from the MK spectral types is not strictly compatible with the values determined by the three other methods. The former determination refers to stars found within the main-sequence band while the three latter determinations refer to the ZAMS. In addition, Table IV lists the previous determinations by Johnson, and Johnson and Morgan. In both of these papers, the authors assumed that interstellar reddening in the cluster was negligible.

Other investigators, including Johnson and Hiltner (1956), Sandage (1957), Johnson (1957), Johnson and Iriarte (1958), and Blaauw (1963) have derived estimates of the color excess and corrected distance modulus of the cluster by the main-sequence fitting

TABLE IV

Summary of reddening and distance determinations

\begin{tabular}{|c|c|c|c|c|c|}
\hline Source & Type & $\bar{E}(B-V)$ & No. & $\overline{V_{0}-M_{v}}$ & No. \\
\hline Johnson (1950) & & $0 \mathrm{~m} \cdot 00$ & - & $10^{\mathrm{m}} \cdot 75$ & \\
\hline Johnson and Morgan (1953) & $U B V$ & 0.00 & & 11.9 & \\
\hline Perry's Method No. 1 & MK & $0.112 \pm 0.022$ & 15 & $11.27 \pm 0.30$ & 15 \\
\hline Perry's Method No. 2 & $U B V$ & $0.100 \pm 0.020$ & 29 & $10.60 \pm 0.26$ & 27 \\
\hline Perry's Method No. 3 & $u v b y-\mathrm{H} \beta$ & $0.129 \pm 0.031$ & 19 & $11.22 \pm 0.24$ & 19 \\
\hline Perry's Method No. 4 & $u v b y-\mathrm{H} \beta$ & $0.107 \pm 0.021$ & 23 & $11.22+0.26$ & 18 \\
\hline Perry's Adopted Mean & & 0.109 & & 11.02 & \\
\hline
\end{tabular}


procedure. Their estimates cluster about the values of $E(B-V)=0^{\mathrm{m}} .11$ and $V_{0}-M_{v}=10^{\mathrm{m}} .8$.

As stated above, the question of cluster membership was investigated by means of $\left(V_{0}, M_{v}\right)$ diagrams. The $U B V$ analysis indicates that 14 of the 45 program stars (Johnson No. 64, $-24^{\circ} 2197,-24^{\circ} 2201,-24^{\circ} 2208$, Johnson No. 65, $-24^{\circ} 2220$, $-24^{\circ} 2221,-24^{\circ} 2226,-24^{\circ} 2235,-24^{\circ} 2240,-24^{\circ} 2246,-24^{\circ} 2250,-24^{\circ} 2253$, and $\left.-24^{\circ} 2254\right)$ are not cluster members. The other three analyses almost always confirm this conclusion.

\section{Acknowledgements}

The writer expresses his thanks to Dr V. M. Blanco, Director of the Cerro Tololo Inter-American Observatory, for permission to use the observatory facilities. He also acknowledges the helpful assistance of $\mathrm{Sr}$ Carlos Bolelli at the telescope. Last but not least, the writer thanks Dr Rudolph Schild for his spectral classifications used in this paper.

This research was supported by a grant from the National Science Foundation.

\section{References}

Blaauw, A.: 1963, in K. Aa. Strand (ed.), Basic Astronomical Data, University of Chicago Press, Chicago, p. 383.

Crawford, D. L. and Strömgren, B.: 1964, Vistas in Astronomy 8, 149.

Crawford, D. L.: 1966, in K. Lodén, L. O. Lodén, and U. Sinnerstad (eds.), 'Spectral Classification and Multicolor Photometry', IAU Symp. 24, 170.

Crawford, D. L.: 1970, in A. Slettebak (ed.), Stellar Rotation, D. Reidel, Dordrecht, Holland, p. 114. Fernie, J. D.: 1965, Astron. J. 70, 575.

Jeffers, H. M. and van den Bos, W. H.: 1963, Publ. Lick Observ. 21, 7.

Johnson, H. L.: 1950, Astrophys. J. 112, 240.

Johnson, H. L.: 1957, Astrophys. J. 126, 121.

Johnson, H. L.: 1963, in K. Aa. Strand (ed.), Basic Astronomical Data, Univesity of Chicago Press, Chicago, p. 204.

Johnson, H. L. and Morgan, W. W.: 1953, Astrophys. J. 117, 313.

Johnson, H. L. and Hiltner, W. A.: 1956, Astrophys. J. 123, 267.

Johnson, H. L. and Iriarte, B.: 1958, Lowell Obs. Bull. 4, 47.

Morgan, W. W., Harris, D. L., and Johnson, H. L.: 1953, Astrophys. J. 118, 92.

Morgan, W. W. and Harris, D. L.: 1956, Vistas in Astronomy 2, 1124.

Perry, C. L. and Hill, G.: 1969, Astron. J. 74, 899.

Sandage, A.: 1957, Astrophys. J. 125, 435.

Schild, R.: private communication.

Strömgren, B.: 1966, in L. Goldberg (ed.), Ann. Rev. Astron. Astrophys. 4.

Walker, G. A. H.: 1965, Astrophys. J. 141, 660. 